\title{
Angiogenese und Vaskularisation beim Tissue Engineering von Fettgewebe
}

\author{
Angiogenesis and Vascularisation in Adipose Tissue Engineering
}

\author{
Autoren \\ J. H. Dolderer ${ }^{1 *}$, F. Medved ${ }^{2 *}$, R. M. Haas $^{2}$, D. I. Siegel-Axel ${ }^{3}$, S. M. Schiller ${ }^{4}$, H.-E Schaller ${ }^{2}$ \\ Institute \\ Die Institutsangaben sind am Ende des Beitrags gelistet
}

\section{Schlüsselwörter \\ - Angiogenese \\ - Vaskularisation \\ - Adipogenese \\ - Gewebezüchtung \\ - Weichteildefekt}

\section{Key words \\ angiogenesis \\ - vascularisation \\ - adipogenesis \\ - tissue engineering \\ - soft-tissue defect}

eingereicht 25.3.2012

akzeptiert $\quad 8.10 .2012$

\section{Bibliografie \\ DOI http://dx.doi.org/ 10.1055/s-0032-1329944 \\ Online-Publikation: 22.1.2013 \\ Handchir Mikrochir Plast Chir \\ 2013; 45: 99-107 \\ (c) Georg Thieme Verlag KG \\ Stuttgart · New York \\ ISSN 0722-1819}

\section{Korrespondenzadresse}

\section{Dr. Jürgen H. Dolderer}

Hochschulzentrum für Plastische Hand- und Wiederherstellungschirurgie

Universitätsklinikum

Regensburg

Franz-Josef-Strauss-Allee 11

93053 Regensburg

drdolderer@hotmail.com

\section{Zusammenfassung}

\section{$\nabla$}

Der heutige Standard für die Deckung und Rekonstruktion größerer Haut-/Weichteildefekte mit freiliegenden Knochen, Nerven oder Gefäßen, wie sie z. B. nach ausgedehnten Tumorresektionen oder komplexen Unfallverletzungen vorkommen, ist der lokale oder freie mikrochirurgische Gewebetransfer. Trotz Einführung und Weiterentwicklung neuer chirurgischer Techniken sowie zahlreicher synthetischer Materialien, ist nach wie vor eine große Anzahl von Limitationen und Komplikationen an der Hebe-/und Empfängerstelle vorhanden. Es besteht daher ein großer Bedarf an Entwicklung neuer Methoden und Materialien, welche einen dauerhaften körpereigenen Weichteilersatz ermöglichen. Ein vielversprechender Therapieansatz ist der Weichteilersatz mit körpereigenem Fettgewebe. Klinische und experimentelle Untersuchungen zur Wiederherstellung von Weichteilen mithilfe körpereigenen Fettgewebes zeigten allerdings bei den freien Fettgewebstransplantationen ohne einen direkten Gefäßanschluss, v.a. im Hinblick auf das Langzeitüberleben des Transplantates, bis dato eher enttäuschende Ergebnisse. Häufig wurde ein Volumenverlust bzw. eine komplette Resorption des Transplantates aufgrund einer insuffizienten Gewebequalität durch ausbleibende Zelldifferenzierung beobachtet. Diese Tatsache verweist auf die besondere Rolle der Unterhaltung und Entwicklung der transplantateigenen Blutversorgung (Vaskularisation und Angiogenese), entscheidend für den Erhalt einer Volumenkonstanz des Gewebes und damit für die Deckung größerer Weichteildefekte. Das schnell wachsende, interdisziplinäre Gebiet des Tissue- Engineerings bietet alternative Lösungsansätze zu den bisherigen Therapiemöglichkeiten. Die Zielsetzung ist hierbei Fettgewebe im

* Die Autoren sind zu gleichen Teilen an der Publikation beteiligt.

\section{Abstract \\ $\nabla$}

The current standard for the reconstruction of large soft tissue defects with exposed bone, nerves or blood vessels, for example after extensive tumor resections, complex injuries, severe burns or infections, is the local or free microsurgical tissue transfer. Despite the development of new surgical techniques and many synthetic materials, there are still a large number of limitations and complications at the donor and recipient site. Thus, in a subset of patients either complete treatment is not possible or poses problems. Therefore, there is a great need for the development of new methods and materials allowing for a permanent replacement with body own soft tissue. A promising therapeutic approach is the soft tissue replacement with autologous adipose tissue. Innovative research on the reconstruction of soft tissue by adipose tissue, and clinical and experimental studies on the long-term survival and transplantation of autologous adipose tissue showed that the free fat tissue graft without direct vascular connection come along with disappointing results. Often a loss of volume or a complete resorption of the graft because of insufficient tissue quality by lack of cell differentiation was observed. This fact points to the special role of the maintenance and development of the graft's blood supply (angiogenesis and vascularization) crucial for maintaining a constant volume of the tissue. The rapidly growing interdisciplinary field of tissue engineering offers alternative solutions to the existing treatment options with the aim to produce autologous adipose tissue, stable in volume in vitro as well as in vivo, which can be transplanted as a permanent tissue replacement to corresponding parts of the body. Numerous studies have demonstrated the important and most critical factor of vascularisation for quality, volume and long-term survival of transplanted newly generated adipose tissue 
Labor sowie zukünftig autolog im Patienten herzustellen, welches als permanenter Gewebeersatz an entsprechende Körperstellen volumenstabil transplantiert werden kann. Zahlreiche Untersuchungen haben die Vaskularisation als bedeutendsten und gleichzeitig kritischsten Faktor für Qualität, Volumen und Langzeitüberleben der transplantierten und komplett neu generierten Fettgewebekonstrukte aufgezeigt. Und obwohl unser Verständnis der regulatorischen Mechanismen der Adipogenese noch sehr eingeschränkt ist, bestehen klare Hinweise darauf, dass die komplexen Abläufe der Zellinteraktionen bei der Proliferation und Differenzierung von Adipozyten in direkter Weise mit der Angiogenese korrelieren.

\section{Einleitung}

$\nabla$

Aktuelle Behandlung von Weichteildefekten

Heutige Therapien für die Rekonstruktion von Weichteildefekten und die Augmentation von Volumendefekten zur Wiederherstellung eines funktionellen, ästhetischen Äußeren beruhen hauptsächlich auf körpereigenen Gewebetransplantationen (lokale, freie Lappenplastiken, dermale Fetttransplantate und freie adipöse Gewebetransplantate) sowie auf artifiziellen Implantaten. Als „Goldstandard“ dafür werden heute myokutane oder adipo- und fasziokutane Lappenplastiken eingesetzt. Bei diesen Lappenplastiken wird zwischen lokalen, gestielten Lappen, welche Transplantationen oder Transpositionen von Weichteilgewebe aus der unmittelbaren Umgebung darstellen, und dem freien mikrovaskulären Gewebetransfer aus entfernten Körperstellen unterschieden [1].

Die Behandlung von Weichteil-/und Volumendefekten mit Lappenplastiken kann allerdings mit nicht geringen Komplikationen verbunden sein. Unter Umständen können funktionelle Defizite im Bereich der Hebe- und/oder Empfängerstelle entstehen. Weiter verbleibt bei allen Lappenplastiken, insbesondere bei freien mikrovaskulären Lappen, das Risiko einer Komplikation an der Gefäßanastomose, von Hämatomen oder Infektionen bzw. eines Lappenverlustes. Weiter besteht das nicht geringe Risiko mehrfacher Folgeoperationen sowie ästhetischer Defizite [2]. Bei ausreichend mit Haut bedeckter, zu augmentierender Körperregion besteht heute die Möglichkeit des Gewebeaufbaus mit biokompatiblen, synthetischen Materialien wie z. B. Silikon oder Gewebeexpandern. Trotz der universalen Verfügbarkeit und Vielfältigkeit hat die Verwendung von Silikonprothesen auch einige nicht unwesentliche Nebenwirkungen und über den Zeitraum von 1992 bis 2005 war der Einsatz von Silikonimplantaten, sowie die Anwendung von injizierbarem Silikon von der Food and Drug Administration (FDA) in Amerika bei gehäuften Kapselkontrakturen, Autoimmunreaktionen und häufigen operativen Revisionen sogar verboten [3]. Die sekundären Fremdkörperreaktionen, wie z.B. eine Kapselfibrose verbunden mit einer Schmerzsymptomatik und unbefriedigendem ästhetischen Ergebnis bei der Verwendung von synthetischen Materialien (z. B. Silikonimplantate), sind hierbei keine immunologischen Frühreaktionen, sondern mit einer Immunreaktion vom Typ IV Spätkomplikationen, welche auch erst Jahre nach Implantation auftreten können [4]. Obwohl durch die heutige Anwendung von texturierten Mammaimplantaten die Inzidenz der Kapselfibrose nach Augmentation gesenkt werden konnte, ist dieses Verfahren bei der Mammaaugmentation zu überdenken, nicht zuletzt da sich in zunehmendem Maße Patientinnen auch den Weichteilgewebsaufbau mit körpereigenem Gewebe wünschen. Ein erster Schritt constructs. Although our understanding of the regulatory mechanisms of adipogenesis is still limited, there are clear indications that the complex sequences of cell interactions in the differentiation and proliferation of adipocytes is directly related to angiogenesis.

in Richtung Brustaufbau durch körpereigenes Gewebe wird in der Plastischen Chirurgie durch die Verwendung von Weichteilgewebetransfers, zum Beispiel im Rahmen von rekonstruktiven Operationen nach Mammakarzinomen, begangen. Bislang wird diese Art der Augmentation vor allem mit gestielten oder freien mikrovaskulären Lappenplastiken ermöglicht. Diese Techniken besitzen jedoch bekanntermaßen ein Komplikationspotenzial sowohl an der Empfängerstelle als auch am Ort der Lappenhebung [2]. Eine vielversprechende Lösung des risikoreduzierten Aufbaus von Weichteildefekten mit Volumenmangel sowie bei der Rekonstruktion von Körperregionen bietet autologes, immunkompatibles Fettgewebe. Dies sollte idealerweise volumenkonstant in ausreichender Menge vorhanden sein und am Ort der Entnahme keinen Funktionsverlust erzeugen. Kürzlich wurden erste Ergebnisse der weiterentwickelten Eigenfetttransplantation mithilfe wasserstrahlassistierter Liposuktion vorgestellt. Hierbei konnte nach volumetrischer Auswertung ein deutlicher Zugewinn des transplantierten Fettes gemessen werden. Der Beobachtungszeitraum betrug allerdings lediglich 6 Monate, sodass noch keine definitive Aussage über die längerfristige Vitalität gemacht werden kann [5]. Eine weitere aussichtsreiche Möglichkeit bietet die Anwendung von „de-novo“ generiertem Fettgewebe. Dies wäre ein großer Vorteil für beispielsweise auch schlanke Patienten, denn die Optionen der Rekonstruktion mittels Eigenfetttransplantation bei schlanker Körperform sind limitiert [6].

\section{Transplantation von Fettgewebe}

Die freie Verpflanzung von Fettgewebe wurde erstmals 1893 von Neuber beschrieben. In seiner Arbeit konnte Neuber den Zusammenhang zwischen einer geringen Transplantatgröße und einer besseren Überlebensfähigkeit des Transplantates aufzeigen [7]. Nur 2 Jahre später gestaltete der Chirurg Vincenz Czerny auf der Basis eines Lipoms eine Brust. Die Resultate waren jedoch bescheiden und die begrenzt guten Ergebnisse ließen sich nur vereinzelt wiederholen. Die Fettgewebsimplantate wurden meist progressiv absorbiert und durch fibröses Gewebe ersetzt, da die Fettzellen wegen ungenügender Versorgung zugrunde gingen und das Proliferationsvermögen der wenigen verbliebenen Fettzellen zu limitiert war [8].

Eine alternative Methode der Fettgewebsaugmentation, die in den letzten 3 Jahrzehnten zunehmend etabliert wurde, ist die Verwendung von mittels Liposuktion gewonnenem autologen Fettgewebe. Klinisch-experimentelle Studien belegen jedoch, dass das applizierte Fettgewebe ein Gemisch aus lebenden und funktionsunfähigen Zellen darstellt. Der Verlust von Gewebemasse in der weiteren Folge ist demnach vorhersehbar [9]. Trotz des teils erheblichen Verlustes an transplantiertem Fettgewebe 
und der damit verbundenen Problematik ist die Fetttransplantation mittels Liposuktion ein weit verbreitetes Verfahren. Die Möglichkeit autologes Gewebe zu transplantieren die Einfachheit des technischen Verfahrens und die geringe Invasivität bietet dem plastischen Chirurgen eine attraktive Methode. In diesem Zusammenhang lag der Fokus vieler Studien auf der Minimierung des Gewebeverlustes nach Injektion. Die untersuchten Methoden reichen vom „Waschen“ mit isotonischer Kochsalzlösung bis hin zum Zufügen von Insulin, Steroiden und vereinzelt auch Wachstumsfaktoren. Entscheidende und über einen längeren Zeitraum konstante statistisch signifikante Unterschiede im verbliebenen Volumen und Größe, je nach Behandlung des Fettgewebes, waren bislang jedoch nicht nachweisbar. Auch die erst vor kurzem veröffentlichten, vielversprechenden Ergebnisse, in welchen mittels eines neuen Verfahrens größere Mengen an Eigenfett mithilfe von wasserstrahlassistierter Liposuktion gewonnen werden, lässt die Frage nach einem langfristigen Massen- und Volumenerhalt offen [5]. Diese bislang mäßigen Resultate bei der Transplantation von körpereigenem Fettgewebe mit erheblicher Resorptionstendenz und starker Neigung zur Fibrosierung sind wahrscheinlich auf eine ungenügende Angiogenese, eine spärliche Ischämietoleranz und auf die starke Stoffwechselaktivität von Adipozyten zurückzuführen [8].

\section{Adipogenese und Angiogenese}

\section{Aufbau und Funktion von Fettgewebe}

Im menschlichen Organismus wird allgemein zwischen 2 Typen von Fettgewebe unterschieden: das braune Fettgewebe, welches vornehmlich bei der Geburt vorhanden ist und eine wichtige Rolle bei der Thermoregulation spielt, sowie das weiße Fettgewebe. Während das braune Fettgewebe mit zunehmendem Alter abnimmt und an Bedeutung verliert, steigt die Menge des im Körper vorhandenen weißen Fettgewebes [10]. Bis vor kurzem wurde die Funktion von weißem Fettgewebe hauptsächlich in einer passiven Rolle bei der langfristigen Speicherung von überschüssiger Energie in Form von Triglyceriden gesehen. Diese Triglyceride können bei Bedarf dem Organismus für die Oxidation wieder zur Verfügung gestellt werden. Weitere Funktionen wurden im Verlauf für die Wärmeregulation, dem mechanischen Schutz sowie bei inflammatorischen Prozessen beschrieben. Entzündliche Vorgänge werden hierbei durch Präadipozyten, welche als „Macrophage-like“ Zellen agieren, vermittelt [11]. Ein entscheidender Wandel in der Betrachtungsweise von weißem Fettgewebe erfolgte in den neunziger Jahren nach der Entdeckung des „Cytokine-like“ Faktors Leptin als wichtigem Bestandteil des Fettgewebes [12]. Mit der Entdeckung des Leptins konnte das weiße Fettgewebe erstmalig als ein endokrines und sezernierendes Organ dargestellt werden, welches entscheidend in die Regulation des Energiehaushaltes integriert ist. Weitere Einflüsse des Leptins wurden zum Beispiel auf das Fortpflanzungssystem, das Immunsystem sowie bei der Angiogenese beschrieben [13]. Neben dem Leptin als Adipozyten-Hormon wurden im Verlauf zahlreiche weitere aus dem Fettgewebe sezernierte, stoffwechselaktive Produkte, die Adipokine, beschrieben. Diese Adipokine beeinflussen u. a. den Fettstoffwechsel, die Blutgerinnung, die Blutdruckregulation sowie den Glucosestoffwechsel und ausschlaggebend die Gefäßneubildung [11,14]. Beispiele für solche angiogenetischen Wachstumsfaktoren mit Einfluss auf die Adipogenese sind Vascular-Endothelial-Growth-Factor (VEGF), Fibroblast-Growth-Factor (FGF), Transforming-Growth-
Factor (TGF) sowie Angiopoietine. Hierbei erfolgt eine parakrine Regulation der Angiogenese von Fettgewebe durch verschiedene Zellen des Fettgewebes, wie Adipose stromal cells (ASC), Präadipozyten und Adipozyten sowie Entzündungszellen [15]. Adipose Stroma cells (ASC) sind hierbei multipotente, mesenchymale Vorläuferzellen, welche ohne weiteres angeregt werden können sich adipogen zu differenzieren. So konnte erst vor kurzem gezeigt werden, dass ASC's funktionelle und phänotypische Überschneidungen mit den Perizyten in der Mikrogefäßstruktur des Fettgewebes haben [16].

Das subkutane weiße Fettgewebe besteht überwiegend aus einer einheitlichen Zellpopulation von Adipozyten. Daneben konnte in mittels Liposuktion gewonnenem humanem Fettgewebe eine heterogene Zellpopulation aus Makrophagen, Mastzellen, Fibroblasten und Fibroblasten-ähnlichen mesenchymalen Vorläuferzellen aufgezeigt werden. Dieser Bestandteil des weißen Fettgewebes, welcher keine reifen Fettzellen enthält, wird auch als stromale Gefäßraktion bezeichnet [17]. Die mechanische Festigkeit von Fettgewebe wird erzielt durch eine Fülle von 3-dimensional konfigurierten, interzellulären Strukturen, welche aus Kollagen oder Laminin bestehen. Über spezifische, an der Zellwand exprimierte Zelladhäsionsmoleküle (z.B. Integrine) werden Zell-Zell Kontakte vermittelt und damit eine sehr hohe interzelluläre Stabilität erreicht [18]. Eine zentrale Rolle spielen im Aufbau des Gewebes auch Kollagenfibrillen. Diese sind nicht nur in Abhängigkeit vom Kollagentyp für die Konsistenz und die mechanischen Eigenschaften verantwortlich, sondern stellen darüber hinaus auch wichtige Zell-Matrix Verbindungen [19]. Das weiße Fettgewebe wird durch ein reiches kapillares Netzwerk versorgt, wobei jede Fettzelle in Kontakt mit wenigstens einer Kapillare ist [20].

\section{Adipogenese}

Adipoblasten sind die frühesten, unipotenten Vorläuferzellen des Fettgewebes. Adipoblasten sind kleine epitheloide Zellen mit einem kleinen perinukleären Zytoplasma [21]. Initial besitzen Adipoblasten keine Fettvakuolen bis sie zu Präadipozyten reifen und sich schließlich zu reifen Fettzellen, den Adipozyten, entwickeln. Vor kurzem konnten Tang et al. nachweisen, dass periendotheliale Perizyten in der Mikrovaskulatur des Fettgewebes die Vorläuferzellen der Adipozyten ausmachen und entscheidend für das Fettgewebewachstum sind. Diese Perizyten sind ähnlich wie glatt-gestreifte Muskelzellen, welche die Endothelzellen in den Blutgefäßen bedecken. Sie exprimieren zum Beispiel Präadipozyten-Marker wie das PPAR- $\gamma$ [22]. Während reife Adipozyten ausdifferenziert und nicht mehr in der Lage sind weiter zu proliferieren, können sich die mesenchymalen Stammzellen und Adipozyten-Vorläuferzellen, die sogenannten Präadipozyten, weiter teilen und differenzieren - abhängig von der Stimulation der Mikroumgebung. Die Adipogenese wird durch viele stimulierende und hemmende Faktoren (exogen und endogen), wie Wachstumsfaktoren und Hormone, beeinflusst [10]. Hierbei ist die Adipogenese eine Abfolge von genetischen Ereignissen. Proteomstudien konnten zeigen, dass Veränderungen in der Expression von mehreren 100 Proteinen an der strukturellen und funktionellen Morphogenese der Adipogenese beteiligt sind. Viele dieser Veränderungen erfolgen dabei auf der transkriptionalen Stufe mit einer geänderten Expression von über 2000 Genen [23]. Als Stimulatoren der Differenzierung sind die Mitglieder zweier Familien von Transkriptionsfaktoren, die CCAAT/vermehrten Bindungsproteine (C/EBP) und die Peroxisom-Proliferations-aktivierten Rezeptoren (PPAR), bekannt. Der 


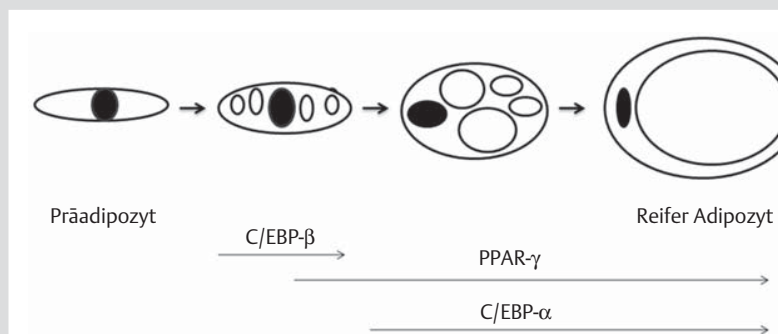

Abb. 1 Transkriptionelle Regulation der Adipozytendifferenzierung. Als Stimulatoren der Differenzierung sind die Mitglieder zweier Familien von Transkriptionsfaktoren, die CCAAT/vermehrten Bindungsproteine (C/EBP) und die Peroxisom-Proliferations-aktivierten Rezeptoren (PPAR) bekannt. Der Beginn der Adipogenese geht mit einer Expression von C/EBP- $\beta$ einher, welcher die Induktion des Transkriptionsfaktor PPAR- $\gamma$ einleitet. Weiter ist für die Induktion der Adipogenese die Expression des Transkriptionsfaktors C/EBP- $\alpha$ notwendig [24].

Beginn der Adipogenese geht mit einer Expression von C/EBP- $\beta$ einher, welcher die Induktion des Transkriptionsfaktor PPAR- $\gamma$ einleitet. Ebenfalls notwendig für die Induktion der Adipogenese ist die Expression des Transkriptionsfaktors C/EBP- $\alpha$ ( $\bullet$ Abb. 1). Ob die Adipozytendifferenzierung initiiert wird, hängt von der Balance der gegensätzlich wirkenden Stimulations- und Hemmungsfaktoren ab, welche mit den Präadipozyten interagieren. Adipozyten sezernieren unterschiedliche Faktoren, wie z. B. Leptin, welches als später Marker der Adipozytendifferenzierung bekannt ist. Diese Wachstums- und Differenzierungssignale werden durch eine Kaskade von intrazellulären Abläufen angeregt. Insulin-like growth factor 1 (IGF-1) ist ein essentieller Regulator der Fettzellformation und ist daher mit Insulin zusammen wichtig für die Adipozytendifferenzierung [24].

\section{Angiogenese im Fettgewebe}

Angiogenese ist in der Literatur häufig mit der Entstehung von Krankheiten, wie zum Beispiel Karzinomen, Arthritis und Psoriasis, assoziiert. Die bedeutende Rolle einer intakten und suffizienten Angiogenese bei der Entstehung und Entwicklung von Gewebe und insbesondere auch von Fettgewebe eines „gesunden“ Organismus gerät dabei oft in den Hintergrund [25].

Jede Fettzelle besitzt Kontakt zu mindestens einer Kapillare. Dies verdeutlicht die relativ hohe Blutperfusionsrate von Fettgewebe im Vergleich zu vielen anderen Organen [26,27]. Die enge und komplexe Querverbindung zwischen der Angiogenese und der Adipogenese konnte in den letzten Jahren genauer aufgezeigt werden. Dabei ließ sich veranschaulichen, dass die Angiogenese hauptsächlich aus Migration, Proliferation und Differenzierung der Endothelzellen besteht, während die Differenzierung der Präadipozyten in reife Adipozyten mit der nachfolgenden Hypertrophie durch Lipideinlagerung die Adipogenese ausmachen [28].

Aus Untersuchungen in Embryos ist bekannt, dass sich bei der Bildung von primitiven Fettorganen das Gefäßbett vor der Adipozytendifferenzierung entwickelt [27]. Das entstehende Gefäßnetzwerk während der embryonale Entwicklung wird durch Vaskulogenese (de-novo Gefäßbildung aus Angioblasten oder Stammzellen) und Angiogenese (Migration, Proliferation und Differenzierung von bereits vorhandenen Endothelzellen) gebildet [29]. Lange Zeit wurde angenommen, dass die postnatale Gefäßneubildung ausschließlich auf dem Prinzip der Angiogenese beruht: ein Prozess, welcher sehr gut bei der Wundhei- lung mit z.B. Verletzung von Fettgewebe durch die Entwicklung von Kapillaren beobachtet werden konnte. Allerdings war der Ursprung der Endothelzellen und der sich entwickelnden Kapillaren lange Zeit unklar [30]. Neue Erkenntnisse zeigen, dass Endothel-Vorläuferzellen im Embryo, wie auch in ischämischem, malignem oder entzündetem Gewebe des Erwachsenen, das Gefäßwachstum bestimmen und sogar therapeutisch für die Stimulation des Gefäßwachstums in ischämischem Gewebe gebraucht werden können. Dieser Prozess wird u.a. deshalb auch als „therapeutische Vaskulogenese“ bezeichnet [25]. Es differenzieren dabei Endothel-Vorläuferzellen zu arteriellen und venösen Endothelzellen, welche wiederum einen einfachen, kapillären Plexus formen. Die werdenden Gefäße bestehen am Anfang nur aus Endothelzellen. Für die weitere Gefäßreifung wird eine Reihe von angio- und arteriogenetischen Faktoren benötigt, damit sich die Endothelzellen zusammenziehen und durch murale Zellen, Perizyten und extrazelluläre Matrix bedeckt werden können. Die Gefäße können dann ausprossen, werden durch glatte Muskelzellen stabilisiert und können folgend von ihren Vorläuferzellen ausdifferenzieren [29].

Fettgewebswachstum erfordert einen kontinuierlichen Umbauprozess des kapillaren Gefäßnetzwerkes. Die Zunahme und das Größenwachstum von Fettgewebe werden durch eine Gefäßneubildung, sowie durch eine Dilatation und Remodellierung von bereits vorhandenen Kapillaren bestimmt. Der Neovaskularisation wird dabei eine Rolle bei der Hyperplasie der Adipozyten zugeschrieben. Für die Hypertrophie der Adipozyten wird die Dilatation und die Remodellierung der existenten Kapillaren verantwortlich gemacht [21]. Darauf aufbauend konnte Brakenhielm zeigen, dass durch die Blockade der Neovaskularisation mit dem Angiogenesehemmer TNP-470 die ernährungsbedingte Fettzunahme verhindert wird [31].

Hämatopoetische Stammzellen wirken direkt und indirekt auf die Angiogenese durch die Differenzierung in Leukozyten und Thrombozyten ein. Der Blutfluss ist ein entscheidender Parameter für das Überleben und den Erhalt der Gefäße. So konnten Carmeliet et al. vor einigen Jahren nachweisen, dass aufgrund insuffizienter angio- und arteriogenetischer Faktoren oder Anwesenheit von angiogenetischen Hemmstoffen die Endothelzellen unausgekleidet, durchlässig und fragil bleiben. In Folge dessen kommt es leicht zu einer Ruptur und Blutung aus dem Gefäß, was wiederum den Blutfluss erheblich reduziert und letztlich in einer Gefäßregression resultiert [25].

Weitere Argumente für die enge Beziehung zwischen Angiogenese und Adipogenese bzw. zwischen Endothel- und Fettzellen konnten durch einen Nachweis der Kommunikation dieser beiden Zelltypen durch parakrine Signalwege, extrazellulärer Matrix und direkte Zell-Zell-Interaktionen gefunden werden $[15,26]$. Humane Präadipozyten und kapilläre Endothelzellen exprimieren $\alpha_{v} \beta_{3}$-Integrin und Plasminogen-Aktivator-Inhibitor-1, welche die Präadipozytenmigration zur Entwicklung eines kapillären Netzwerkes steuern, um wiederum die Koordination der Entwicklung beider Gewebe am selben Platz zu sichern [32]. Seit längerem ist bekannt, dass Vorläuferzellen des Fettgewebes die Wundheilung unterstützen und ischämisches Gewebe, einschließlich des Myokardgewebes, revaskularisieren können [33]. Diese Feststellung unterstützt, dass das Fettgewebe angiogenetische Moleküle produziert. Die Regulation der Angiogenese im Fettgewebe geschieht durch multiple Faktoren. So bewirken verschiedene Zellpopulationen im Fettgewebe, wie Präadipozyten, Adipozyten, Fettstromazellen sowie Entzündungszellen, die Produktion von multiplen angiogenetischen Faktoren (u.a. 
VEGF). Diese angiogenetischen Faktoren regulieren zusammen mit den ebenfalls exprimierten Inhibitoren, wie z.B. Endostatin, Thrombospondin 1 (TSP-1), die Angiogenese im Fettgewebe. Es ergibt sich aus diesem Zusammenspiel verschiedener Faktoren ein angiogenetischer Synergismus [34]. Angiogenetische Wachstumsfaktoren mit Einfluss auf die Adipogenese besitzen die Fähigkeit, Neovaskularisation in vivo zu induzieren. Sie können grob in 4 Klassen aufgeteilt werden: 1. Vascular-endothelialgrowth-factor (VEGF), 2. Fibroblast-growth-factor (FGF), 3. platelet-derived-growth-factors (PDGF) und 4. Transforminggrowth-factors (TGF). VEGF, auch als VEGFA bezeichnet, gehört mit placental-growth-factor (PLGF), VEGFB, VEGFC und VEGFD zu einer Genfamilie. VEGF ist ein endothelspezifisches Mitogen, das von speziellen Zellen sezerniert wird (z.B. retinale Zellen, Myokardzellen und Präadipozyten). Bei der Transkription eines einzigen Gens können 4 Isoformen VEGF exprimiert werden, wobei VEGF $_{165}$ am häufigsten vorkommt. Wichtig für die endotheliale Zellproliferation, Zellmigration und das Überleben der Endothelzellen ist die Interaktion von VEGF mit den Rezeptoren VEGFR-1 und VEGFR-2. Hausmann et al. beschreiben VEGF als den entscheidenden Faktor bei der Angiogenese von Fettgewebe, da VEGF die Anordnung unreifer Gefäße initiiert. In Untersuchungen mit Mäuseembryos konnte gezeigt werden, dass die Ausschaltung eines einzigen VEGF Allels deren Letalität bedingte [35]. Auf das Gefäßsystem wirken weiter 4 Unterformen der fibroblast growth factors ein: FGF-1 (acidic oder aFGF), FGF-2 (basic oder bFGF), FGF-4 und FGF-5. Alle zeigen mitogene Effekte auf Endothelzellen: bFGF stimuliert z. B. die Endothelzellproliferation und -migration und wird von den Endothelzellen produziert um Perizyten zu rekrutieren [36]. PDGF ist ein potenter Stimulator für das Wachstum und die Motilität der Fibroblasten sowie der glatten Muskelzellen; er wirkt weiter auch auf Endothelzellen und Neurone ein. Es konnte nachgewiesen werden, dass die Abwesenheit von PDGF-B zu Hämorrhagien führt bedingt durch die verminderte, stabilisierende Umhüllung von Gefäßen mit Perizyten [37]. TGF und seine Rezeptoren sind wichtige Regulatoren der Endothelzellproliferation sowie notwendig bei der Etablierung und Beständigkeit der Gefäßintegrität. Der Effekt von TGF auf die Angiogenese beruht am ehesten auf der Rekrutierung von Makrophagen und Fibroblasten, die angiogenetische Faktoren sezernieren. TGF fungiert wahrscheinlich weiterhin als Stabilisator neu geformter Gefäße durch Rekrutierung von glatten Muskelzellen und Perizyten sowie durch Förderung ihrer Proliferation. TGF induziert eine PDGF-Expression durch Endothelzellen sowie VEGF und bFGF-Expression in glatten Muskelzellen [25]. Es werden in wachsendem Fettgewebe jedoch auch hohe Mengen an Angiogeneseinhibitoren, v.a. Thrombospondin 1 (TSP-1) und Endostatin, produziert. Dabei wird angenommen, dass diese Inhibitoren erforderlich sind, nachdem sich das neu-entstandene Fettgewebe stabilisiert hat, um ein weiteres Gefäßwachstum zu begrenzen. Entsprechend findet sich auch eine Verminderung der TSP-1-Expression in Präadipozyten und eine erhöhte TSP-1-Expression in differenzierten Adipozyten [15]. Eine entscheidende Rolle bei der vaskulären Umbildung vermittelt durch verminderte Expression in wachsendem Fettgewebe spielen Angiopoietine. Soweit bekannt existieren 5 Angiopoetinproteine: Ang- $1,-2,-3,-4$ und -5 . Angiopoietin-1 wird dabei von periendothelialen Zellen exprimiert, während die anderen Angiopoietine z.B. von Zellen der umbilikalen Venen oder fetalen Alveolarzellen exprimiert werden. Ang-1 initiiert und stabilisiert mittels TIE-2-Rezeptoren das Gefäßwachstum. Eine geringere Menge von Ang-1 erlaubt den

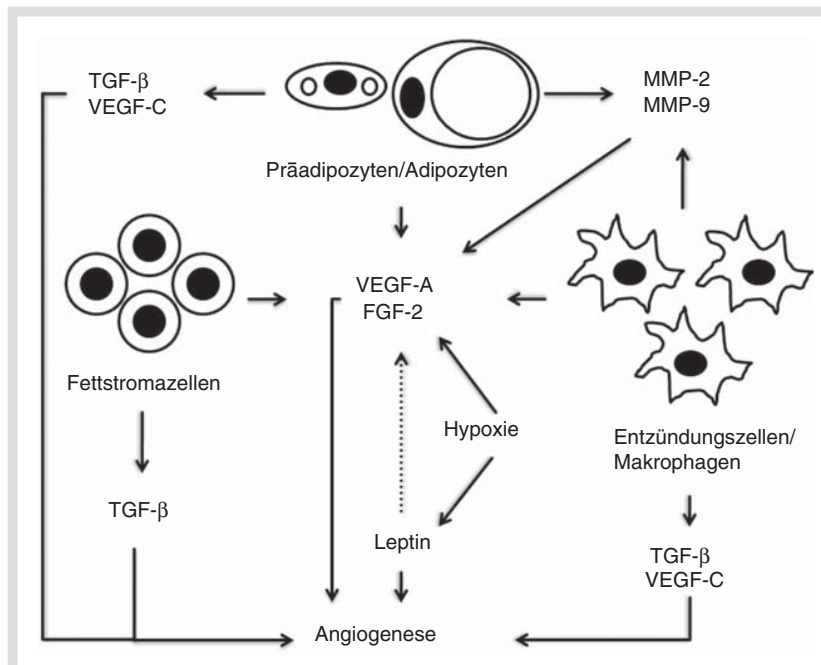

Abb. 2 Direkte und Indirekte Regulation der Angiogenese durch Präadipozyten/Adipozyten, Fettstromazellen und Entzündungszellen. Im Zentrum VEGF-A als der entscheidene Faktor der Angiogenese induziert durch Zellen des Fettgewebes [15].

wandständigen Zellen, darunter den Perizyten und den glatten Gefäßmuskelzellen, sich von dem Blutgefäß zu lösen, um dadurch eine erhöhte Exposition und Sensitivität von Endothelzellen für andere Angiogenesefaktoren wie VEGF zu schaffen [38]. Gewebehypoxie kann ebenfalls zu einer vermehrten Expression von VEGF und Leptin führen. Matrixmetalloproteinasen (MMP) aus dem Fettgewebe bewirken eine erhöhte Bioverfügbarkeit von angiogenetischen Faktoren durch die Freisetzung von Matrix-gebundenem VEGF [26]. Die Ergebnisse veranschaulichen, dass diese Faktoren, vermittelt durch die Regulation der Angiogenese, auch einen wichtigen Einfluss auf die Adipogenese haben ( $\odot$ Abb. 2).

\section{Tissue Engineering von Fettgewebe}

$\nabla$

Das fortschrittliche Verfahren des Tissue Engineering beruht darauf, lebende Zellen eines Organismus als 3-dimensionales Gewebekonstrukt zu kultivieren. In den letzten Jahren konnten im Bereich des Tissue Engineerings grundlegende Erkenntnisse hinzu gewonnen werden und im Hinblick auf Gewebezüchtung mit der Möglichkeit der Weichteilrekonstruktionen eröffnen sich dabei wichtige Lösungsansätze. Die Chance der de-novo Produktion von ausreichend vaskularisiertem, reifem, autologem Fettgewebe als Alternative zu Implantaten und komplizierten Gewebetransfers stellt einen solchen Ansatz dar [9]. Die vaskuläre Versorgung wird als der entscheidende Aspekt angesehen, welcher die Größe, Aufrechterhaltung und Qualität eines durch Tissue Engineering generierten Konstruktes limitiert. Die bisher erfolgreich hergestellten Tissue Engineering Produkte umfassen vornehmlich dünne Gewebestrukturen wie künstlich gezüchtete Haut, Herzklappen und großlumige Gefäße, welche bis zur Revaskularisierung durch die Empfängerstelle mittels Diffusion überleben können. Aktuell existiert kein klinisch anwendbares „tissue engineered“ Konstrukt mit einer eigenständigen, intrinsischen Vaskularisierung und der Möglichkeit eines direkten Gefäßanschlusses an ein extrinsisches Empfängergefäß. Bei der Herstellung großer Gewebevolumina bereitet sowohl die Notwendigkeit einer makroskopischen Zirkulation, als 
auch der Bedarf eines mikrovaskulären Netzwerkes weiterhin Schwierigkeiten. So zeigen sich Fettzellen mit mehr als $200 \mu \mathrm{m}$ Entfernung von einem Blutgefäß aufgrund ihrer eingeschränkten Versorgung durch Diffusion nekrotisch oder metabolisch untätig [8]. Transplantierbare Gewebekonstrukte mit Eignung für die rekonstruktive Chirurgie besitzen ein zu großes Volumen um ausreichende Oxygenierung und Ernährung allein durch Diffusion zu bewerkstelligen. Kürzlich durchgeführte Untersuchungen in Tiermodellen beschreiben Versuche mit einer intrinsischen Blutversorgung ebenso wie mit entsprechender Veränderung der Mikroumgebung. Allerdings wurden die Stadien einer klinischen Anwendbarkeit bisher noch nicht erreicht [27,39,40]. Etliche bisherige Untersuchungen verdeutlichen die komplexe Verbindung zwischen der Entstehung von Fettgewebe und der Angiogenese. Aus diesem Grund sollten beide Prozesse bei der Gewebezüchtung von klinisch größenrelevantem Fettgewebe beachtet werden $[27,39,40]$. Die Generierung von vaskularisiertem Gewebe mit klinisch relevanter Größe erfordert eine Quelle (intrinsische Blutgefäße) und eine vordefinierte Struktur (Matrix) mit Durchlässigkeit für Nährstoffe. Diese Matrix stellt letztlich auch ein Sammelbecken von Wachstumsfaktoren dar, welche für die Angioinduktion verantwortlich gemacht werden. Vor kurzem wurde die Implantation von Gefäßschleifen in Wachstumskammern in situ untersucht. Die Blutversorgung und Gefäßneubildung wird hier von Beginn an beibehalten und es ergibt sich eine gute Möglichkeit für die Produktion größerer Gewebevolumina $[39,41]$. Die Entstehung eines neugewachsenen Gewebekonstruktes, welches auf der Verwendung einer Gefäßschleife basiert, wurde von Mian beschrieben [42]. Es glückte der Versuch, spontan und im Tiermodell, Lappen aus unspezifischem Granulationsgewebe heranzüchten, indem eine arteriovenöse Gefäßschleife (AV-Shunt) gemeinsam mit einer Kollagenmatrix einem Kammermodell zugeführt wurde. Tanaka et al. untersuchten neben dem AV-Shunt die Formierung eines arteriovenösen Gefäßbündels im Hinblick auf eine Gefäßneubildung und erreichbare Gewebevolumen in einem Kammermodell. Sie beschrieben eine maximale Gewebeproduktion im AV-ShuntModell. Jedoch implementierten die Ergebnisse auch, dass das arterio-venöse Gefäßbündel als Gefäßstiel für klinische Zwecke besser geeignet ist. Es zeigte sich weiter, dass eine proangiogenetische Kommunikation mit der Umgebung durch Perforationen in der Kammerwand positiv auf das Gewebewachstum wirkt [41]. Für die Entstehung von großvolumigen 3-dimensionalen vaskularisierten Gewebekonstrukten sind Baugerüste (Scaffolds) von zusätzlicher Wichtigkeit. Diese Baugerüste sollen das wachsende Gewebe stabilisieren und ihm eine 3-dimensionale Form vorgeben. Auch sollen die Scaffolds eine stärkere Proliferation von Adipozyten und Endothelzellen ermöglichen [43]. Die vermehrte Expression von Rezeptoren sowie unterschiedlicher Wachstumsfaktoren (z.B. FGF-2, VEGF) im Kammermodell bedingen eine Zunahme des Gewebewachstums und der Angiogenese [34]. Neben dem Einfluss verwendeter Biomaterialien auf die Neovaskularisation konnten in vorangegangenen Untersuchungen die hypoxischen Bedingungen in der Kammer als entscheidende und steuernde Faktoren identifiziert werden [44]. Trotz der Abwesenheit einer extrazellulären Matrix wird in den meisten Verfahren des Tissue Engineerings entweder eine synthetische oder biologische Matrix zur Unterstützung und Beschleunigung des Gewebwachstums herangezogen. In diesen Fällen tritt Fibrin in die Kammer ein und stellt ein Gerüst für die folgende Gefäß- und Gewebeneubildung bereit. Eine Alternative zum AV-Shunt ist der ligierte Gefäßstiel. Die spontane Bildung von Anastomosen zwischen benachbarter Arterie und Vene innerhalb weniger Tage sowie die Etablierung eines konstanten Blutflusses hierüber konnten bereits aufgezeigt werden. Es konnte nachgewiesen werden, dass unter Anwendung eines ligierten Gefäßstiels sowohl mehr organisiertes Gewebe als auch eine größere Dichte an gebildeten Kapillargefäße des gezüchteten Konstruktes auftraten [41]. Aktuelle Untersuchungen zu Generierung von 3-dimensionalem, größenkonstantem Fettgewebe mittels Tissue engineering zeigen, dass in geschlossenen Wachstumskammern unter der Verwendung von AV-Shunts ein größeres Volumen vaskularisierten Gewebes entsteht als bei ligiertem Gefäßstiel. Der höhere Durchflussdruck innerhalb des Venentransplantates und der Vene selbst könnte eine stärkere Extravasation des Fibrins als bei den ligierten Gefäßstielen bewirken [45]. Es wird daher postuliert, dass die erhöhte Fibrinmenge zu einer gesteigerten Vaskularisation und damit zu einem stärkerem Gewebewachstum führt. Allerdings zeigte sich das Gewebe, welches mit ligiertem Gefäßstiel generiert wurde, im Vergleich zu dem Gewebe, welches durch einen arterio-venösen Shunt ernährt wurde, besser organisiert, weniger gereift und durch eine höhere Dichte an neu gebildeten Kapillargefäßen gekennzeichnet [41]. In folgenden Untersuchungen mit perforierter Wachstumskammer zeigte sich bei ligiertem Gefäßstiel mehr Gewebsbildung als bei der Verwendung von AV-Shunts. Bereits zuvor hatte sich ein Volumenvorteil des Gewebes zugunsten der mit Perforationen durchsetzten Kammern gezeigt, welcher auf das gesteigerte Einsprießen von Gefäßen zurückgeführt wurde [46]. Ebenso scheint der durch die Perforationen ermöglichte Fibrineinfluss von Bedeutung zu sein. Diese Beobachtung unterstreicht die Wichtigkeit der Fibrinablagerung als Wegbereiter für die Gefäßaussprossung im Rahmen der Neovaskularisation [47]. In dem Modell der perforierten Wachstumskammer mit ligiertem Gefäßstiel findet eine zunehmende Angiogenese auch außerhalb der Kammer statt. Mithilfe der Perforationen kommt es schließlich zu einem vermehrten Einwachsen von angiogenetischen Zellen in die Kammer und zur Stimulation der in der Kammer vorhanden Zellen. Die Perforationen ermöglichen demnach offensichtlich ein sehr viel schnelleres Einwachsen und Auffüllen des Kammervolumens mit angiofibrotischem Gewebe. Das Vorhandensein und schnelle Einwachsen einer proangiogenetischen Matrix sowie eine Veränderung der Scherkräfte bedingten einen positiven Einfluss dieses Kammermodells auf die Angiogense. Auch findet eine viel schnellere Remodellierung in den perforierten Kammern mit ligiertem Gefäßstiel statt, was die Gefäßdichte und Reifung beeinflusst. Allerdings scheint die Hypoxie die entscheidende Rolle bei dem Gewebewachstum zu spielen. Auf das Kammermodell bezogen bedingt a.e. die Isolation innerhalb der Kammer eine hypoxische Umgebung, in welcher die proliferierenden Zellen, einschließlich Endothelzellen, Perizyten und myofibroblastische Zellen, in großer Anzahl schon innerhalb der ersten Woche beobachtet werden können. Dies legt eine Stimulation von zellulärer und angiogenetischer Proliferation induziert durch Hypoxie nahe [47]. Die Ergebnisse in der Kombination eines ligierten Gefäßstiels mit einer perforierten Wachstumskammer lassen ein großes Potenzial für die Neovaskularisation und das Gewebewachstum erkennen. Vor kurzem veröffentlichte Untersuchungen unter Verwendung eines Gefäßstiel mit adhärentem vaskularisierten Fettgewebe in Kombination mit einer perforierten Wachstumskammer verdeutlichen, dass es möglich ist, auf diese Weise größere Mengen stabiles, transplantierbares Fettgewebe zu generieren [48]. Das Gewebewachstum erfolgt entlang eines vorbestehenden Gefäß- 

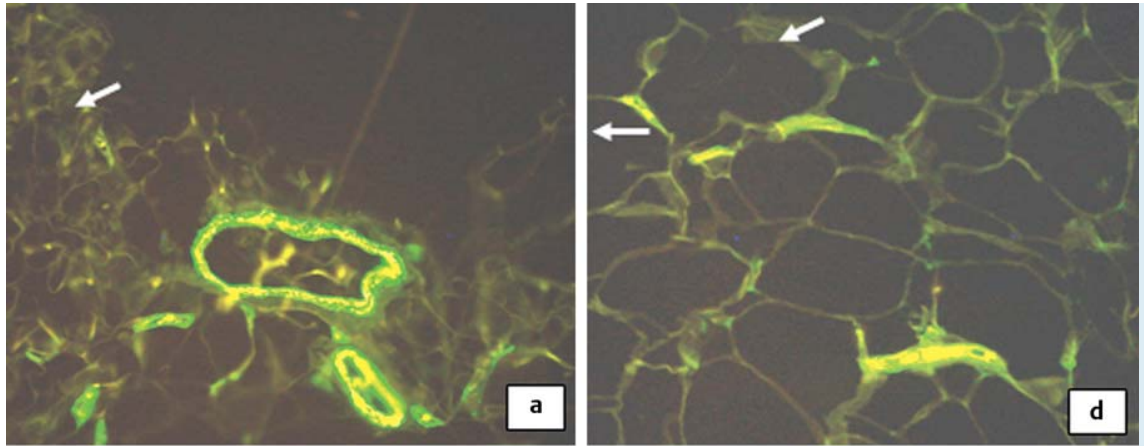

Abb. 3 De-novo erzeugtes Fettgewebe der Ratte mithilfe einer perforierten Wachstumskammer $\mathbf{a}-\mathbf{c}$ und ohne Wachstumskammer $\mathbf{d}-\mathbf{f}$, mit immunhistochemischem Nachweis einer vermehrten Angiogenese innerhalb des Fettgewebes unter Verwendung einer Wachstumskammer. a und d: Nachweis des für glatte Gefäßmuskelzellen charakteristischen $\alpha$-Aktins, b und e: des für Endothelzellen charakteristischen Oberflächenmarkers PECAM-1 (CD31) sowie $\mathbf{c}$ und $\mathbf{f}$ : des von Endothelzellen gebildeten von Willebrand Faktors (vWF) (mit freundlicher Genehmigung Dolderer
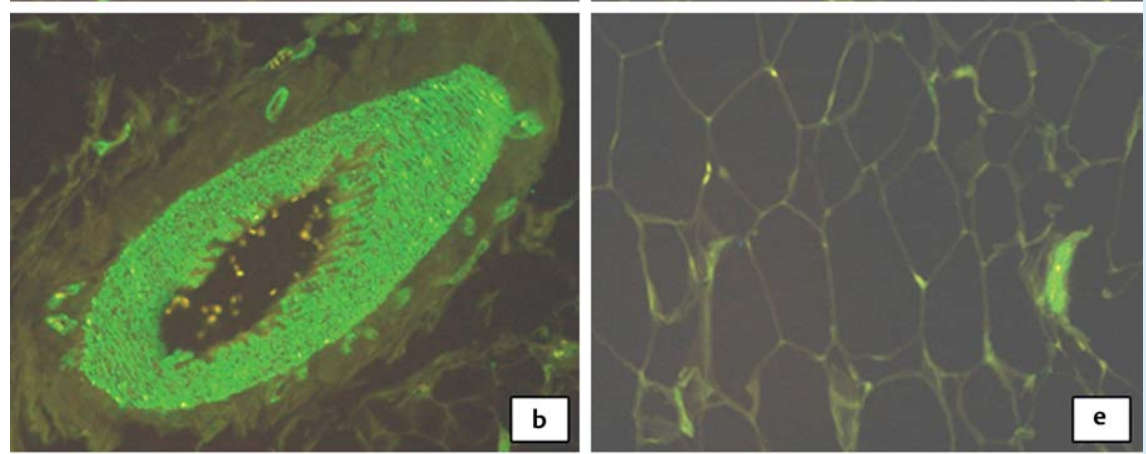
et al., unveröffentlichte Ergebnisse).
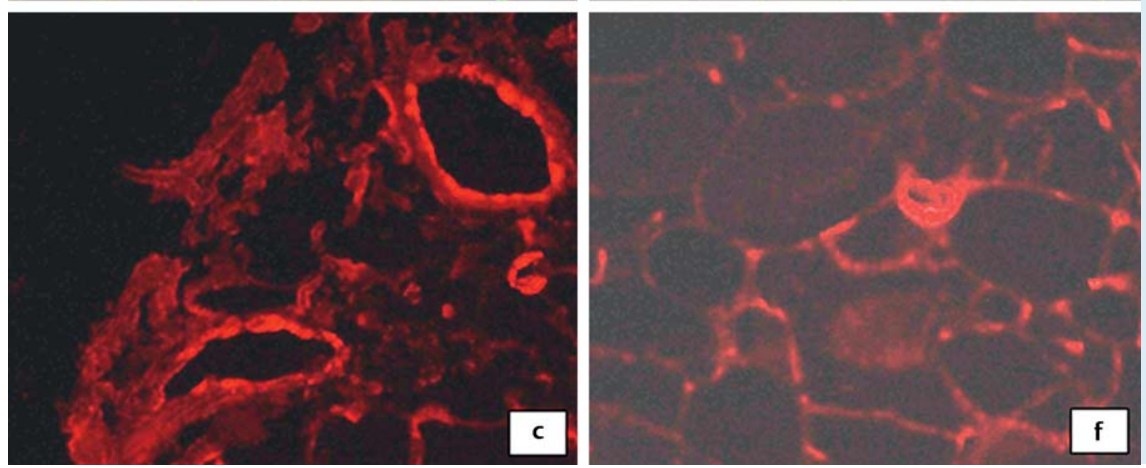

stiels, der selbst expandiert, die proliferierenden Adipozyten ernährt sowie gleichzeitig eine Transplantation des Konstruktes an entfernte Körperstellen erlaubt. Die Kammer fungiert als inkompressibler Schutzraum und ermöglicht die Ausbildung eines vom umliegenden Gewebe weitgehend separierten Milieus [47].

\section{Langzeitstabilität und Volumenkonstanz von durch} Tissue-engineering de-novo generiertem Fettgewebe Das durch das Tissue-engineering erzielte, vermehrte Fettgewebswachstum ist hauptsächlich auf eine Steigerung der Angiogenese zurückzuführen ( $\bullet$ Abb. 3). Dies ist entscheidend für die Generierung von klinisch relevanten Mengen an Fettgewebe sowie für dessen Langzeitstabilität. In entsprechenden Untersuchungen konnte die zeitliche Stabilität der durch Tissue-engineering mittels ligierten Gefäßstiels und perforierten Wachstumskammer gewonnenen Fettgewebskonstrukte auch nach Transplantation bewiesen werden. Jüngst konnte ebenfalls aufgezeigt werden, dass die Stabilität und die Form über einen größeren Zeitraum auch außerhalb der Kammer intakt ist. Ein andauerndes, vermehrtes Fettgewebswachstum nach Entfernung der Wachstumskammer konnte nicht mehr gesehen werden [49]. Vorangegangene Analysen von Walton et al. untersuchten Fettgewebskonstrukte in einem Modell mit dem Wachstumsfaktor bFGF und Matrigel. Allerdings ist Matrigel ein potentielles Malignitätskriterium und es konnten keine Aussagen im Hinblick auf die Transplantation und Stabilität des Gewebeverban- des nach Entfernung aus den umhüllenden Silikonschichten getroffen werden [40]. Die Forschungsarbeiten mit ligiertem Gefäßstiel und perforierter Wachstumskammer verdeutlichen, dass große Volumina reifen Fettgewebes in vivo generiert werden können, welche auf einem intrinsischen Gefäßstiel basieren [50]. Bisher sind keine weiteren Wachstumskammermodelle bekannt, in welchen klinisch relevante Volumina an vaskularisiertem und transplantablem Fettgewebe gezüchtet werden konnten. Limitationen und Einschränkungen ergeben sich allerdings aus der Größe der Kammer und den verwendeten Biomaterialen.

Die Angiogenese ist dementsprechend ein wichtiger Bestandteil des Tissue-engineering von Fettgewebe und kann die Züchtung von Fettgewebe auch ohne Einsatz exogener angiogenetischer und adipogenetischer Wachstumsfaktoren steigern. Zukünftig sollte die therapeutische Einflussnahme bei der Angiogenese auf zell-basierten Ansätzen wie Isolierung, Differenzierung und Expandierung ausgewählter und adäquater Vorläuferzellen oder Stammzellpopulation zur Gefäßformation und -reifung beruhen sowie auf Verwendung der Zusammenhänge bekannter molekularbiologischer Regelkreise für die Stimulation der Angiogenese und damit auch der Adipogenese. Die Zugabe von angiogenetischen Stimulatoren und Hemmstoffen, welche nötig sind um reife Gefäße zu formen, sollte auf ein Minimum beschränkt werden. Aufgrund der Ähnlichkeit zu den Präparationen der bislang am Menschen durchgeführten Lappenplastiken ist die klinische 
Eignung der „de-novo“ Fettgewebszüchtung unter Verwendung eines ligierten Gefäßstiels und einer perforierten Wachstumskammer gut denkbar. Da keine ex-vivo Zellen eingebracht werden, ist ein klinischer Einsatz durch das Medizinproduktegesetzt klar geregelt und mit der Möglichkeit der de-novo Generierung größerer Volumina an vaskularisiertem Fettgewebe sowie nach Erhalt entsprechender Langzeitergebnisse ist ein klinischer Einsatz in etwa 3 Jahren vorstellbar. Entsprechend könnten in Zukunft körpereigene Fettgewebslappen bei der Wiederherstellung von Weichteildefekten, wie z. B. nach schweren Unfallverletzungen, ausgedehnten Infektionen und Tumoroperationen oder bei Brustrekonstruktionen ohne künstliche Implantate eingesetzt werden. Weiter stellt diese Methodik des Tissue-engineerings eine minimal-invasive Technik bei der Produktion von vaskularisierten und größenkonstanten Fettgewebskonstrukten dar. Insgesamt ist dies unserer Meinung nach eine Bereicherung für die Regenerative Medizin und die Rekonstruktive Chirurgie: insbesondere für die Generierung eines autologen Weichteilersatzes.

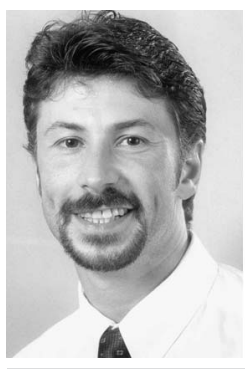

Dr. med. Jürgen $\mathrm{H}$. Dolderer

Studium der Humanmedizin an der Johann Wolfgang Goethe-Universität in Frankfurt/Main. Promotion 1998 mit „Summa cum laude“. 1997-2001 Assistenzarzt in der Klinik für Chirurgie des Nordwestkrankenhauses Frankfurt (Prof. Dr. H. Bockhorn). 2001-2004 DFG-Forschungsstipendiat am Bernard O`Brien Institute for Microsurgery sowie klinisches Fellowship Plastische und Mikrochirurgie am St. Vincent Hospital (Prof. Dr. Wayne Morrison), University of Melbourne, Australien. 2004-2005 Klinik für Plastische-, Hand- und Rekonstruktive Chirurgie, Verbrennungszentrum, BG-Unfallklinik Ludwighafen (Prof. Dr. G. Germann), Universität Heidelberg. 2006 Facharzt für Chirurgie. 2007 Abteilung für Plastische-, Wiederherstellungs-, Ästhetische und Mund-, Kiefer- und Gesichts-Chirurgie am Klinikum Mittelbaden in Rastatt (Dr. Dr. R. Herr). Von 2007 bis 2012 Klinik für Plastische-, Hand-, Rekonstruktive- und Verbrennungschirurgie, BG-Unfallklinik Tübingen, Eberhard-Karls-Universität Tübingen (Prof. Dr. H. E. Schaller). 2009 Facharzt für Plastische und Ästhetische Chirurgie. 2010 Zusatzbezeichnung Handchirurgie. Seit Mai 2012 Oberarzt am Hochschulzentrum für Plastische, Rekonstruktive, Hand- und Ästhetische Chirurgie des Universitätsklinikums Regensburg tätig.

Interessenkonflikt: Nein

\section{Institute}

'Hochschulzentrum für Plastische, Hand- und Wiederherstellungschirurgie, Universitätsklinikum Regensburg, Regensburg

${ }^{2}$ Klinik für Hand, Plastische, Rekonstruktive und Verbrennungschirurgie, BG-Unfallklinik, Eberhard Karls Universität Tübingen, Tübingen

${ }^{3}$ Klinik für Innere Medizin, Endokrinologie, Diabetologie, Angiologie, Nephrologie und Klinische Chemie, Eberhard Karls Universität Tübingen, Tübingen ${ }^{4}$ Freiburg Institute for Advanced Studies (FRIAS), Albert-Ludwigs Universität Freiburg, Freiburg

\section{Literatur}

1 Patrick CW Jr. Tissue engineering strategies for adipose tissue repair. The Anatomical record 2001; 263: 361-366

2 Henriksen TF, Holmich LR, Fryzek JP et al. Incidence and severity of short-term complications after breast augmentation: results from a nationwide breast implant registry. Annals of plastic surgery 2003; 51: 531-539

3 Kessler DA. The basis of the FDA's decision on breast implants. The New England journal of medicine 1992; 326: 1713-1715

4 Stock W, Wolf $K$. Capsule fibrosis in silicone implants. Langenbecks Archiv fur Chirurgie 1986; 369: 303-308

5 Herold C, Ueberreiter $K$, Cromme $F$ et al. Is there a need for intrapectoral injection in autologous fat transplantation to the breast? - An MRI volumetric study. Handchirurgie, Mikrochirurgie, plastische Chirurgie: Organ der Deutschsprachigen Arbeitsgemeinschaft fur Handchirurgie: Organ der Deutschsprachigen Arbeitsgemeinschaft fur Mikrochirurgie der Peripheren Nerven und Gefasse 2011; 43: 119-124

6 Maxwell GP, Gabriel A. Possible future development of implants and breast augmentation. Clinics in plastic surgery 2009; 36: 167-172

7 Neuber G. Fettransplantation. Verh Dtsch Ges Chir 1893; 22: 66

8 Beahm EK, Walton RL, Patrick CW Jr. Progress in adipose tissue construct development. Clinics in plastic surgery 2003; 30: 547-558

9 Tanzi MC, Fare S. Adipose tissue engineering: state of the art, recent advances and innovative approaches. Expert review of medical devices 2009; 6: 533-551

10 Choi JH, Gimble JM, Lee $K$ et al. Adipose tissue engineering for soft tissue regeneration. Tissue engineering Part B, Reviews 2010; 16: 413-426

11 Trayhurn P, Beattie JH. Physiological role of adipose tissue: white adipose tissue as an endocrine and secretory organ. The Proceedings of the Nutrition Society 2001; 60: 329-339

12 Zhang $Y$, Proenca $R$, Maffei $M$ et al. Positional cloning of the mouse obese gene and its human homologue. Nature 1994; 372: 425-432

13 Bouloumie A, Drexler HC, Lafontan $M$ et al. Leptin, the product of Ob gene, promotes angiogenesis. Circulation research 1998; 83: 1059-1066

14 Trayhurn P, Wood IS. Adipokines: inflammation and the pleiotropic role of white adipose tissue. The British journal of nutrition 2004; 92: 347-355

15 Cao Y. Angiogenesis modulates adipogenesis and obesity. The Journal of clinical investigation 2007; 117: 2362-2368

16 Rajashekhar G, Traktuev DO, Roell WC et al. IFATS collection: Adipose stromal cell differentiation is reduced by endothelial cell contact and paracrine communication: role of canonical Wnt signaling. Stem Cells 2008; 26: 2674-2681

17 Zuk PA, Zhu M, Mizuno $H$ et al. Multilineage cells from human adipose tissue: implications for cell-based therapies. Tissue engineering 2001; 7: 211-228

18 Nakajima I, Yamaguchi T, Ozutsumi $K$ et al. Adipose tissue extracellular matrix: newly organized by adipocytes during differentiation. Differentiation; research in biological diversity 1998; 63: 193-200

19 Pierleoni C, Verdenelli F, Castellucci $M$ et al. Fibronectins and basal lamina molecules expression in human subcutaneous white adipose tissue. European journal of histochemistry: EJH 1998; 42: 183-188

20 Bulow J. Measurement of adipose tissue blood flow. Methods Mol Biol 2001; 155: 281-293

21 Hausman GJ, Kauffman RG. The histology of developing porcine adipose tissue. Journal of animal science 1986; 63: 642-658

22 Tang $W$, Zeve $D$, Suh JM et al. White fat progenitor cells reside in the adipose vasculature. Science 2008; 322: 583-586

23 Guo X, Liao K. Analysis of gene expression profile during 3T3-L1 preadipocyte differentiation. Gene 2000; 251: 45-53

24 Niemelä SMS, Sarkanen JR, Ashammakhi N. Adipose Tissue and Adipocyte Differentiation: Molecular and Cellular Aspects and Tissue Engineering Applications. In: N Ashammakhi RR, Chiellini F, Hrsg. Topics in Tissue Engineering 2008; 1-26

25 Carmeliet $P$. Angiogenesis in health and disease. Nature medicine 2003; 9: 653-660

26 Bouloumie A, Lolmede K, Sengenes $C$ et al. Angiogenesis in adipose tissue. Annales d'endocrinologie 2002; 63: 91-95

27 Crandall DL, Hausman GJ, Kral JG. A review of the microcirculation of adipose tissue: anatomic, metabolic, and angiogenic perspectives. Microcirculation 1997; 4: 211-232

28 Dvorak HF. Angiogenesis: update 2005. Journal of thrombosis and haemostasis. JTH 2005; 3: 1835-1842

29 Jain RK. Molecular regulation of vessel maturation. Nature medicine 2003; 9: 685-693 
30 Lokmic Z. Tissue engineering, vascular biology [Dissertation]. Melbourne, Australia: University of Melbourne; 2005; 1-47

31 Brakenhielm E, Cao R, Gao B et al. Angiogenesis inhibitor, TNP-470, prevents diet-induced and genetic obesity in mice. Circulation research 2004; 94: 1579-1588

32 Crandall DL, Busler DE, McHendry-Rinde B et al. Autocrine regulation of human preadipocyte migration by plasminogen activator inhibitor-1. The Journal of clinical endocrinology and metabolism 2000; 85: 2609-2614

33 Vineberg AM, Shanks J, Pifarre $R$ et al. Myocardial Revascularization by Omental Graft without Pedicle: Experimental Background and Report on 25 Cases Followed 6 to 16 Months. The Journal of thoracic and cardiovascular surgery 1965; 49: 103-129

34 Baluk P, McDonald DM. Markers for microscopic imaging of lymphangiogenesis and angiogenesis. Annals of the New York Academy of Sciences 2008; 1131: 1-12

35 Hausman GJ, Richardson RL. Adipose tissue angiogenesis. Journal of animal science 2004; 82: 925-934

36 Yla-Herttuala S, Alitalo K. Gene transfer as a tool to induce therapeutic vascular growth. Nature medicine 2003; 9: 694-701

37 Cleaver O, Melton DA. Endothelial signaling during development. Nature medicine 2003; 9: 661-668

38 Stoeltzing 0 , Ahmad SA, Liu W et al. Angiopoietin-1 inhibits tumour growth and ascites formation in a murine model of peritoneal carcinomatosis. British journal of cancer 2002; 87: 1182-1187

39 Cronin KJ, Messina A, Knight KR et al. New murine model of spontaneous autologous tissue engineering, combining an arteriovenous pedicle with matrix materials. Plastic and reconstructive surgery 2004; 113: 260-269

40 Walton RL, Beahm EK, Wu L. De novo adipose formation in a vascularized engineered construct. Microsurgery 2004; 24: 378-384

41 Tanaka Y, Sung KC, Tsutsumi A et al. Tissue engineering skin flaps: which vascular carrier, arteriovenous shunt loop or arteriovenous bundle, has more potential for angiogenesis and tissue generation? Plastic and reconstructive surgery 2003; 112: 1636-1644
42 Mian R, Morrison WA, Hurley JV et al. Formation of new tissue from an arteriovenous loop in the absence of added extracellular matrix. Tissue engineering 2000; 6: 595-603

43 Chiu YC, Cheng MH, Uriel S et al. Materials for engineering vascularized adipose tissue. Journal of tissue viability $2011 ; 20$ : 37-48

44 Hofer SO, Mitchell GM, Penington AJ et al. The use of pimonidazole to characterise hypoxia in the internal environment of an in vivo tissue engineering chamber. Br J Plast Surg 2005; 58: 1104-1114

45 Dolderer JH, Kehrer A, Schiller SM et al. De-novo generation of vascularized tissue using different configurations of vascular pedicles in perforated and closed chambers. Wien Med Wochenschr 2010; 160: 139-146

46 Tanaka Y, Sung KC, Fumimoto $M$ et al. Prefabricated engineered skin flap using an arteriovenous vascular bundle as a vascular carrier in rabbits. Plast Reconstr Surg 2006; 117: 1860-1875

47 Lokmic Z, Stillaert F, Morrison WA et al. An arteriovenous loop in a protected space generates a permanent, highly vascular, tissueengineered construct. The FASEB journal: official publication of the Federation of American Societies for Experimental Biology 2007; 21: 511-522

48 Dolderer JH, Abberton KM, Thompson EW et al. Spontaneous large volume adipose tissue generation from a vascularized pedicled fat flap inside a chamber space. Tissue engineering 2007; 13: 673-681

49 Dolderer JH, Thompson EW, Slavin J et al. Long-term stability of adipose tissue generated from a vascularized pedicled fat flap inside a chamber. Plastic and reconstructive surgery 2011; 127: 2283-2292

50 Findlay MW, Dolderer JH, Trost $N$ et al. Tissue-Engineered Breast Reconstruction: Bridging the Gap toward Large-Volume Tissue Engineering in Humans. Plastic and reconstructive surgery 2011; 128: 1206-1215 